\title{
Using DTMB-Based Passive Radar for Small Unmanned Aerial Vehicle Detection
}

\author{
Huijie Zhu $\mathbb{D}^{1,2}$ Lijun Wang, ${ }^{1,2}$ and Mingqian Liu $^{3}$ \\ ${ }^{1}$ Science and Technology on Communication Information Security Control Laboratory, Jiaxing 314033, China \\ ${ }^{2}$ The 36th Research Institute of China Electronics Technology Group Corporation, Jiaxing 314033, China \\ ${ }^{3}$ State Key Laboratory of Integrated Service Networks, Xidian University, Xi'an 710071, China
}

Correspondence should be addressed to Huijie Zhu; zhuhuijie@zju.edu.cn

Received 3 March 2021; Revised 28 March 2021; Accepted 20 April 2021; Published 6 May 2021

Academic Editor: Liangtian Wan

Copyright (c) 2021 Huijie Zhu et al. This is an open access article distributed under the Creative Commons Attribution License, which permits unrestricted use, distribution, and reproduction in any medium, provided the original work is properly cited.

There is inevitable polarization angle deviation between the target echo signal and the direct path signal of illuminator of opportunity (IO) in passive radar. In order to investigate the potential performance loss in target detection induced by the random deviation, small unmanned aerial vehicle (UAV) detection experiments with digital television terrestrial multimedia broadcasting- (DTMB-) based passive radar are conducted in this paper. Experimental results show that the polarization angles of the clutter signal and target echo signal are inconsistent. When the polarization diversity technology is used to suppress the clutter signal, the processing performance of the target echo signal may be reduced. On the premise that clutter is effectively suppressed by the processing algorithm, polarization synthesis can maximize the target echo signal processing gain. The effectiveness of the target localization algorithm combining time difference of arrival (TDOA) and direction of arrival (DOA) is also verified with polarization diversity reception in this paper.

\section{Introduction}

Passive radar is a type of special bistatic or multistatic radar which exploits the existing electromagnetic signals in space as illuminators of opportunity (IOs) and realizes target detection and localization through passive reception. As its features of no dedicated transmitter and no frequency assignment, passive radar has attracted wide attention from academia and industry. FM radio $[1,2]$, digital TV $[3,4]$, mobile communications [5, 6], Wi-Fi [7, 8], WiMAX [9-11], satellite communications $[12,13]$, and many other IOs have been exploited for passive radar, which makes it achieve remarkable development over the years.

The IOs are not purposely designed for target detection; as a result, passive radar needs complicated signal processing to ensure its performance. However, the cost of signal processing has been greatly reduced with the development of high-performance computing technology. Furthermore, the development of software-defined radio technology makes passive radar be developed towards software-defined radar. The passive radar researchers only need to focus on algo- rithms rather than hardware platforms, which greatly promotes the iterative evolution of passive radar processing algorithms. The signal processing of passive radar has approached its ultimate performance at present, and it is urgent to find new ways to improve the performance of passive radar.

In recent years, polarization diversity is generally considered a feasible way to further improve the passive radar performance [1, 14-18]. However, most studies focused on realizing clutter suppression by taking advantage of polarization diversity technology. While the clutter signal is effectively suppressed, the detection performance of the target echo signal may be limited by the polarization angle deviation between the clutter signal and the echo signal.

In order to verify the performance of the polarization diversity-based echo signal detection, field experiments with dual-polarization reception are carried out in this paper. The digital television terrestrial multimedia broadcasting (DTMB) signal is exploited as the IO signal while the unmanned aerial vehicle (UAV) of DJI Mavic 2 is selected as the target. DTMB is an international terrestrial high- 
definition television broadcasting standard led by China. The bandwidth of the DTMB signal is about $8 \mathrm{MHz}$ while the transmission power is usually between 300 and 1000 watts.

The rest of this paper is organized as follows. Section 2 introduces the signal model for passive radar in detail. Section 3 analyzes the polarization diversity for clutter suppression and target detection, together with the polarization-induced problem. Section 4 discusses the experimental process and results. Finally, conclusions and future work are summarized in Section 5.

\section{Signal Model for Passive Radar}

2.1. Signal Model for Receiving Channels. A passive radar system generally consists of two synchronous receiving channels, which are called the reference channel and surveillance channel, respectively. The received signal $x_{r}(t)$ of the reference channel can be described as

$$
x_{r}(t)=C_{r} \cdot s(t)+\sum_{i=1}^{L} C_{r, i} \cdot s\left(t-\tau_{i}\right)+w_{r}(t)
$$

where $s(t)$ is the pure signal transmitted by the IO source, $C_{r}$ is the amplitude, $L$ is the number of multipath reflection, $C_{r, i}$ and $\tau_{i}$ are the amplitude and time delay of the $i_{\text {th }}$ multipath, respectively, and $w_{r}(t)$ is the noise in the reference channel. $x_{r}(t)$ usually has a high signal-to-noise ratio (SNR) as the reference antenna is in the direction of the IO when the passive radar is in operation. Especially for the digital modulated signal, we can reconstruct the pure signal $s(t)$ from $x_{r}(t)$ by means of demodulation and remodulation [4]; (1) can then be simply rewritten as

$$
x_{r}(t) \approx C_{r} \cdot s(t)
$$

The received signal $x_{s}(t)$ of the surveillance channel can be written as

$$
\begin{aligned}
x_{s}(t)= & \sum_{i=0}^{N_{c}} C_{s, i} \cdot s\left(t-\tau_{s, i}\right)+\sum_{i=N_{c}+1}^{N_{c}+N_{s}} C_{s, i} \cdot s\left(t-\tau_{s, i}\right)+\sum_{i=1}^{N_{m}} C_{m, i} \cdot s \\
& \cdot\left(t-\tau_{m, i}\right) e^{j 2 \pi f_{i} t}+w_{s}(t) ;
\end{aligned}
$$

in (3), $C_{s, i}$ and $\tau_{s, i}$ are the amplitude and time delay of the $i_{\text {th }}$ stationary object echo (including multipath), respectively, and $i=0$ is for the direct path signal (DPS) received from the side lobe of the surveillance antenna. $C_{m, i}, \tau_{m, i}$, and $f_{i}$ are the amplitude, time delay, and Doppler frequency of the $i_{\text {th }}$ moving target echo, respectively. $N_{c}$ is the number of clutter echoes (excluding the DPS), $N_{s}$ is the number of stationary targets, $N_{m}$ is the number of moving targets, and $w_{s}(t)$ is the noise in the surveillance channel.

2.2. Signal Model for Clutter Suppression. The energy of the target echo is far weaker than that of clutter in passive radar. Clutter suppression is crucial for the operation of passive radar as it may mask target echoes. The least-squares (LS) matrix solution, sometimes referred to as the extensive cancelation algorithm (ECA) or direct matrix inversion (DMI), is a widely used method as its inherently parallel algorithm structure that can be perfectly accelerated by hardware implementation $[19,20]$. According to (3), the signal model for the surveillance channel can be written in a discrete form after sampling as

$$
\begin{aligned}
x_{s}(n) & =x_{c}(n)+x_{t}(n)+w_{s}(n) \\
& =\sum_{i=0}^{K_{c}} C_{s, i} \cdot s(n-i)+x_{t}(n)+w_{s}(n),
\end{aligned}
$$

where $x_{c}(n), x_{t}(n)$, and $w_{s}(n)$ are the discrete form of clutter echoes, target echoes (both stationary and moving), and noise, respectively. $s(n)$ is the discrete form of $s(t), K_{c}$ is the maximum integer number corresponding to a signal delay in samples of the clutter echoes, and (4) can be rewritten in a compact matrix form as

$$
\mathrm{X}_{s}=\mathrm{S} \cdot \mathrm{C}_{s}+\mathrm{X}_{t}+\mathrm{W}_{s}
$$

in (5),

$$
\begin{aligned}
\mathrm{X}_{s} & =\left[\begin{array}{lccc}
x_{s}(n) x_{s}(n+1) & \cdots & x_{s}(n+N)
\end{array}\right]^{T}, \\
\mathrm{~S} & =\left[\begin{array}{cccc}
s(n) & s(n-1) & \cdots & s\left(n-K_{c}\right) \\
s(n+1) & s(n) & \cdots & s\left(n-K_{c}+1\right) \\
\vdots & \vdots & \ddots & \vdots \\
s(n+N) & s(n+N-1) & \cdots & s\left(n+N-K_{c}\right)
\end{array}\right], \\
\mathrm{C}_{s} & =\left[\begin{array}{lllll}
C_{s, 0} & C_{s, 1} & \cdots & C_{s, K_{c}}
\end{array}\right]^{T}, \\
\mathrm{X}_{t} & =\left[\begin{array}{llll}
x_{t}(n) & x_{t}(n+1) & \cdots & x_{t}(n+N)
\end{array}\right]^{T}, \\
\mathrm{~W}_{s} & =\left[\begin{array}{llll}
w_{s}(n) & w_{s}(n+1) & \cdots & w_{s}(n+N)
\end{array}\right]^{T},
\end{aligned}
$$

where $N$ is the number of available samples and $[\cdot]^{T}$ indicates matrix transposition.

The ECA-based solution is first to solve the following optimization problem:

$$
\min _{C} J=\left\|\mathrm{X}_{s}-\mathrm{S} \cdot \mathrm{C}\right\|^{2}
$$

The solution of (11) can be found by calculating the pseudoinverse of $\mathbf{S}$ :

$$
\mathrm{C}=\left(\mathrm{S}^{H} \mathrm{~S}\right)^{-1} \mathrm{~S}^{H} \mathrm{X}_{s}
$$

where $[\cdot]^{H}$ indicates matrix conjugate transposition. The calculated coefficient vector $\mathbf{C}$ represents estimated values of the clutter amplitudes for successive delays of the reference signal. Then, the clutter suppression can be expressed as

$$
\mathrm{X}_{\mathrm{rem}}=\mathrm{X}_{s}-\mathrm{S} \cdot \mathrm{C} \text {; }
$$

in (13), 


$$
\mathrm{X}_{\mathrm{rem}}=\left[\begin{array}{llll}
x_{\text {rem }}(n) & x_{\text {rem }}(n+1) & \cdots & x_{\text {rem }}(n+N)
\end{array}\right]^{T}
$$

is the residual target echo signal vector after clutter cancellation.

For the operation of passive radar, a substitution of $x_{r}(t)$ for $s(t)$ is usually valid due to the high SNR. As a result, the clutter suppression in practice is performed as

$$
\mathrm{X}_{\mathrm{rem}}=\mathrm{X}_{s}-\mathrm{X}_{r}\left(\mathrm{X}_{r}^{H} \mathrm{X}_{r}\right)^{-1} \mathrm{X}_{r}^{H} \mathrm{X}_{s}
$$

in (15),

$$
\mathrm{X}_{r}=\left[\begin{array}{cccc}
x_{r}(n) & x_{r}(n-1) & \cdots & x_{r}\left(n-K_{c}\right) \\
x_{r}(n+1) & x_{r}(n) & \cdots & x_{r}\left(n-K_{c}+1\right) \\
\vdots & \vdots & \ddots & \vdots \\
x_{r}(n+N) & x_{r}(n+N-1) & \cdots & x_{r}\left(n+N-K_{c}\right)
\end{array}\right],
$$

where $x_{r}(n)$ is the discrete form of $x_{r}(t)$.

2.3. Signal Model for Target Detection and Localization. Target detection in passive radar is achieved by applying a cross-ambiguity function (CAF) to the residual target echo and the time-Doppler shifted visions of the reference signal. The calculation process is defined as

$$
\psi(l, k)=\sum_{n=0}^{N-1} x_{\mathrm{rem}}(n) x_{r}^{*}(n-l) \mathrm{e}^{-j 2 \pi n k / N}
$$

by maximizing the CAF, the bistatic parameter pair of the strongest target echo signal can be estimated as

$$
\left(l_{t}, k_{t}\right)=\underset{l, k}{\arg \max }|\psi(l, k)| ;
$$

then, the estimated time delay $\tau_{t}$ and Doppler-shift $f_{t}$ can be derived as

$$
\begin{gathered}
\tau_{t}=\frac{l_{t}}{f_{s}}, \\
f_{t}=k_{t} \frac{f_{s}}{N} .
\end{gathered}
$$

The time difference of arrival (TDOA) measurement method for finding a target position has been widely used in the presence of multiple transmitters or multiple receivers [21-24]. The main target localization method used for bistatic passive radar is based on the measurements of bistatic range and direction of arrival (DOA) [25-31]. Different from the beamforming-based DOA estimation technique, a 2-D interferometric approach is exploited to estimate the DOA of the target echo in this paper. Though the beamforming technique can enhance the signal-to-disturbance ratio, the

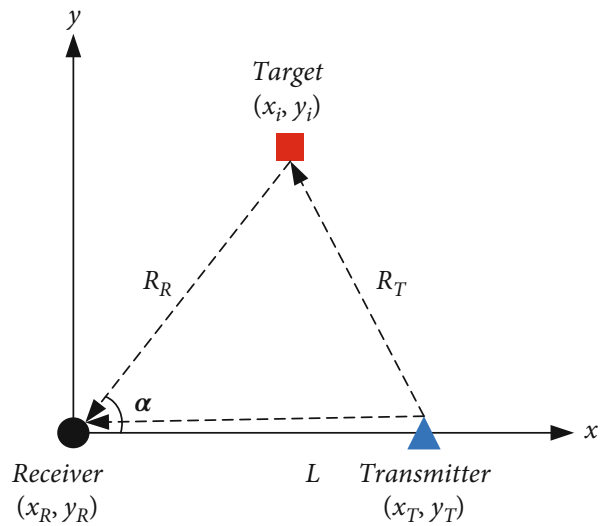

FIGURE 1: Bistatic plane within a 2-D coordinate system.

interferometric approach is simple and sufficient to verify the superiority of polarization diversity.

According to (17) and (18), the CAF results of the $M$ surveillance channels can be described as

$$
\begin{aligned}
\psi\left(l_{t}, k_{t}\right) & =\left[\begin{array}{llll}
\psi_{0}\left(l_{t}, k_{t}\right) & \psi_{1}\left(l_{t}, k_{t}\right) & \cdots & \psi_{M-1}\left(l_{t}, k_{t}\right)
\end{array}\right]^{T} \\
& =\mathrm{a}(\mathbf{d}, \alpha) \sum_{n=0}^{N-1} x_{\mathrm{rem}}(n) x_{r}^{*}\left(n-l_{t}\right) \mathrm{e}^{-j 2 \pi n k_{t} / N},
\end{aligned}
$$

where $\mathbf{a}(\mathbf{d}, \alpha)=\left[a_{0}\left(d_{0}, \alpha\right), a_{1}\left(d_{1}, \alpha\right), \cdots, a_{M-1}\left(d_{M-1}, \alpha\right)\right]^{T}$ is the steering vector of the antenna array, $d_{m}(m=0,1, \cdots$, $M-1)$ is the distance difference between the $m_{\text {th }}$ and the reference antenna elements, and $\alpha$ is the DOA of the target echo. Therefore, DOA estimation based on the $\mathrm{CAF}$ results is completely equivalent to the interferometer direction finding.

Figure 1 shows the bistatic plane within a 2-D coordinate system, where the coordinates of receiver, transmitter, and target are $\left(x_{R}, y_{R}\right),\left(x_{T}, y_{T}\right)$, and $\left(x_{i}, y_{i}\right)$, respectively. Without loss of generality, we can set $\left(x_{R}, y_{R}\right)$ as $(0,0)$ for simplification. Then, the baseline $L$ can be calculated from the known positions of both receiver and transmitter as

$$
L=\sqrt{x_{T}^{2}+y_{T}^{2}}
$$

the range between the transmitter and the target $R_{T}$ can be derived from the unknown position of the target and the known transmitter as

$$
R_{T}=\sqrt{\left(x_{T}-x_{i}\right)^{2}+\left(y_{T}-y_{i}\right)^{2}}
$$

similarly, $R_{R}$ shown in Figure 1 can be described as

$$
R_{R}=\sqrt{x_{i}^{2}+y_{i}^{2}}
$$

with the estimations of time delay $\tau_{t}$ and target echo DOA $\alpha$, additional equations can be obtained as 


$$
\left\{\begin{array}{l}
c \cdot \tau_{t}=R_{T}+R_{R}-L, \\
\tan \alpha=\frac{y_{i}}{x_{i}}
\end{array}\right.
$$

where $c$ is the speed of propagation.

The closed-form localization result can be realized by solving (22), (23), and (24) as

$$
\left\{\begin{array}{l}
x_{i}=\frac{c \cdot \tau_{t} \cdot\left(2 L+c \cdot \tau_{t}\right) \cos \alpha}{2\left(L+c \cdot \tau_{t}\right)-2 x_{T}-2 y_{T} \sin \alpha}, \\
y_{i}=\frac{c \cdot \tau_{t} \cdot\left(2 L+c \cdot \tau_{t}\right) \sin \alpha}{2\left(L+c \cdot \tau_{t}\right)-2 x_{T}-2 y_{T} \sin \alpha}
\end{array}\right.
$$

\section{Polarization Diversity and Problem Analysis}

There is inevitable polarization angle deviation between the echo signal and the incident signal. The effects of polarization have not been taken into account in the signal model above. When the polarization angle is present, (3) can be rewritten as

$$
\begin{aligned}
x_{s}(t)= & \sum_{i=0}^{N_{c}} C_{s, i} \cdot s\left(t-\tau_{s, i}\right) \cdot \cos \theta_{s, i}+\sum_{i=N_{c}+1}^{N_{c}+N_{s}} C_{s, i} \cdot s\left(t-\tau_{s, i}\right) \cdot \cos \theta_{s, i} \\
& +\sum_{i=1}^{N_{m}} C_{m, i} \cdot s\left(t-\tau_{m, i}\right) e^{j 2 \pi f_{i} t} \cdot \cos \theta_{m, i}+w_{s}(t),
\end{aligned}
$$

where $\theta_{s, i}$ and $\theta_{m, i}$ are the polarization angles of the $i_{\text {th }}$ stationary object echo and the $i_{\text {th }}$ moving object echo, respectively, and they are parameters that we can control under polarization diversity reception.

Most of the IOs for passive radar are transmitted by horizontal or vertical polarization antennas. Assume we use an orthogonal dual-polarized antenna as the surveillance antenna, the received signal vector can be described as

$$
\mathrm{X}(n)=\left[\begin{array}{c}
x_{0^{\circ}}(n) \\
x_{90^{\circ}}(n)
\end{array}\right]=\left[\begin{array}{c}
\cos \theta \\
\sin \theta \cdot e^{j \varphi}
\end{array}\right] x_{s}(n),
$$

where $x_{0^{\circ}}(n)$ is one output of the dual-polarized antenna and $x_{90^{\circ}}(n)$ is the other, $\theta$ is the polarization angle determined by the antenna, and $\varphi$ is the phase difference between the two orthogonal channels. After polarization diversity reception, the arbitrary polarization angle can be realized by polarization synthesis as follows:

$$
\begin{aligned}
\widehat{x}_{s}(n, \vartheta, \phi) & =\left[\begin{array}{ll}
\cos \vartheta & \sin \vartheta \cdot e^{j \phi}
\end{array}\right]^{*}\left[\begin{array}{c}
x_{0^{\circ}}(n) \\
x_{90^{\circ}}(n)
\end{array}\right] \\
& =\left[\begin{array}{ll}
\cos \vartheta & \sin \vartheta \cdot e^{-j \phi}
\end{array}\right]\left[\begin{array}{c}
\cos \theta \\
\sin \theta \cdot e^{j \varphi}
\end{array}\right] x_{s}(n) \\
& =\left(\begin{array}{ll}
\cos \vartheta \cos \theta+\sin \vartheta \sin \theta \cdot e^{j(\varphi-\phi)}
\end{array}\right) \cdot x_{s}(n)
\end{aligned}
$$

in (28), $[\cdot]^{*}$ indicates the conjugate operation. By substituting $\phi=\varphi$ in (28), we obtain

$$
\widehat{x}_{s}(n, \vartheta)=\cos (\theta-\vartheta) \cdot x_{s}(n)
$$

which means that the polarization angle has changed. We can then rewrite (15) as

$$
\mathrm{X}_{\mathrm{rem}}(\vartheta, \phi)=\widehat{\mathrm{X}}_{s}-\mathrm{X}_{r}\left(\mathrm{X}_{r}^{H} \mathrm{X}_{r}\right)^{-1} \mathrm{X}_{r}^{H} \widehat{\mathrm{X}}_{s} .
$$

The essence of clutter suppression by taking advantage of polarization diversity technology can be expressed as

$$
\min _{\vartheta, \phi} J=\left\|\mathrm{X}_{\mathrm{rem}}(\vartheta, \phi)\right\|^{2}
$$

the optimal polarization synthesis parameter pair for clutter suppression can be described as

$$
\left(\theta_{c}, \varphi_{c}\right)=\underset{\vartheta, \phi}{\arg \min }\left\|\mathrm{X}_{\mathrm{rem}}(\vartheta, \phi)\right\|^{2}
$$

On the premise that clutter can be effectively suppressed by a processing algorithm, target detection in passive radar will only depend on the CAF operation. The optimal polarization synthesis parameter pair for the $\left(l_{t}, k_{t}\right)$ target detection can be obtained as

$$
\begin{aligned}
\left(\theta_{t}, \varphi_{t}\right) & =\underset{\vartheta, \phi}{\arg \max }\left|\widehat{\psi}\left(l_{t}, k_{t}, \vartheta, \phi\right)\right| \\
& =\underset{\vartheta, \phi}{\arg \max }\left|\sum_{n=0}^{N-1} x_{\text {rem }}(n, \vartheta, \phi) x_{r}^{*}\left(n-l_{t}\right) e^{-j 2 \pi n k_{t} / N}\right|,
\end{aligned}
$$

where $x_{\text {rem }}(n, \vartheta, \phi)$ is the element of $\mathbf{X}_{\text {rem }}(\vartheta, \phi)$.

Therefore, when the parameter pairs of $\left(\theta_{c}, \varphi_{c}\right)$ and $\left(\theta_{t}\right.$, $\left.\varphi_{t}\right)$ are inconsistent, clutter suppression based on the polarization diversity technology will result in performance loss for the target detection.

\section{Experimental Analysis}

In order to verify the theoretical analysis above, we conduct an experimental system which consists of a reference antenna, surveillance antenna, software-defined radio (SDR) receiver, and heterogeneous computing platform. The experimental antenna system is shown in Figure 2. It is mainly composed of a vertical polarization antenna and a dual-polarized antenna.

4.1. Reference Signal Reconstruction. It can be clearly seen from Figure 2 that the DTMB transmitting antenna is visible to the reference antenna. Therefore, the reference signal has a high SNR. Further, reference signal reconstruction is adopted to remove the inevitable multipath and noise. The channel decoding and coding operations are omitted in our processing due to the high SNR, and the whole reference signal reconstruction process is given in Figure 3. 


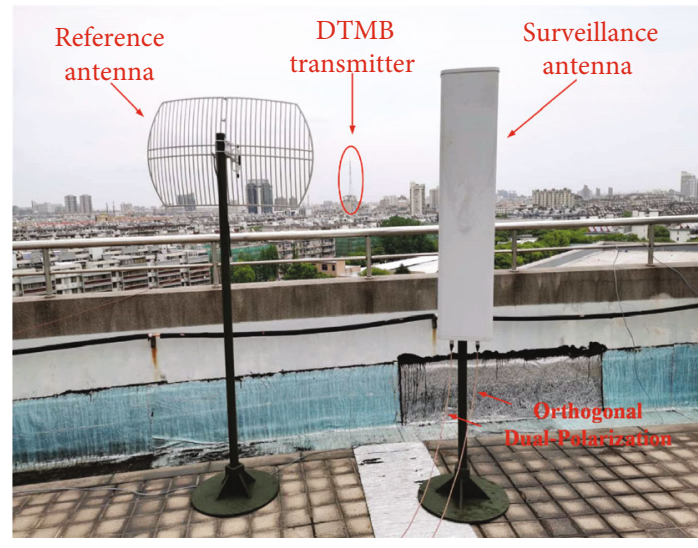

FIGURE 2: The experimental antenna system.

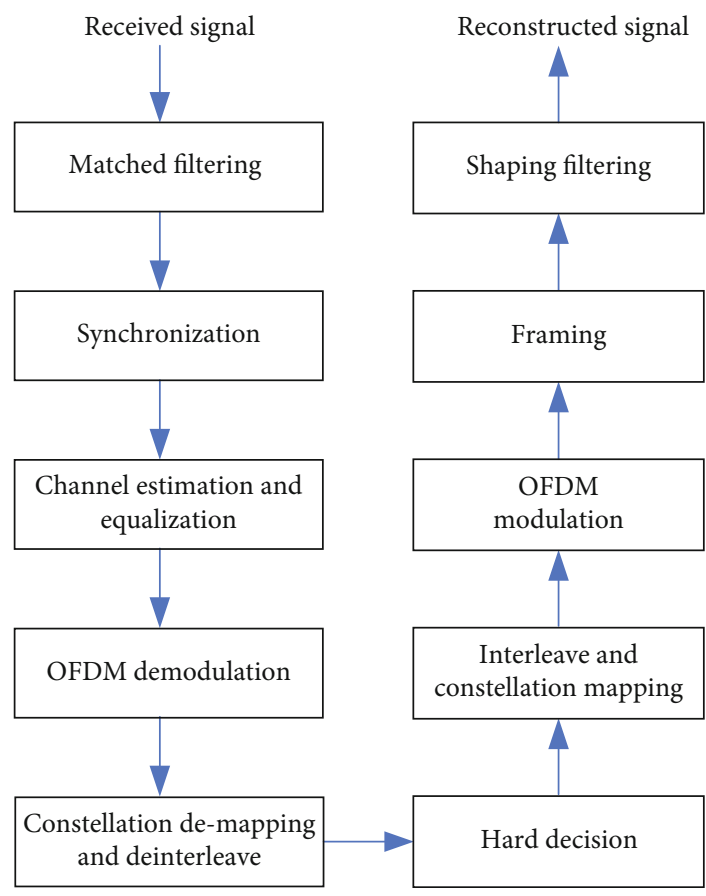

FIgURE 3: Reference signal reconstruction process.

Matched filtering is first performed to reduce intersymbol interference for the received signal. Then, synchronization, channel estimation and equalization, OFDM demodulation, and other operations are carried out in turn.

A multichannel synchronous SDR receiver is used for signal acquisition, which can turn the radio frequency and sampling rate flexibly. The carrier frequency of the DTMB signal exploited in our experiments is $754 \mathrm{MHz}$, and the used sampling rate is $30.24 \mathrm{MHz}$, which means that the oversampling rate is 4 . The duration of the reference signal used for demodulation is $0.1 \mathrm{~s}$. Figure 4 shows the obtained oneframe constellation after OFDM demodulation. According to the DTMB standard, the frame body of the DTMB signal consists of two different types of symbols, the system information (SI) symbol and the streaming data symbol. The SI symbol is mapped to the BPSK constellation fixedly while the streaming data symbol can be mapped to one of the

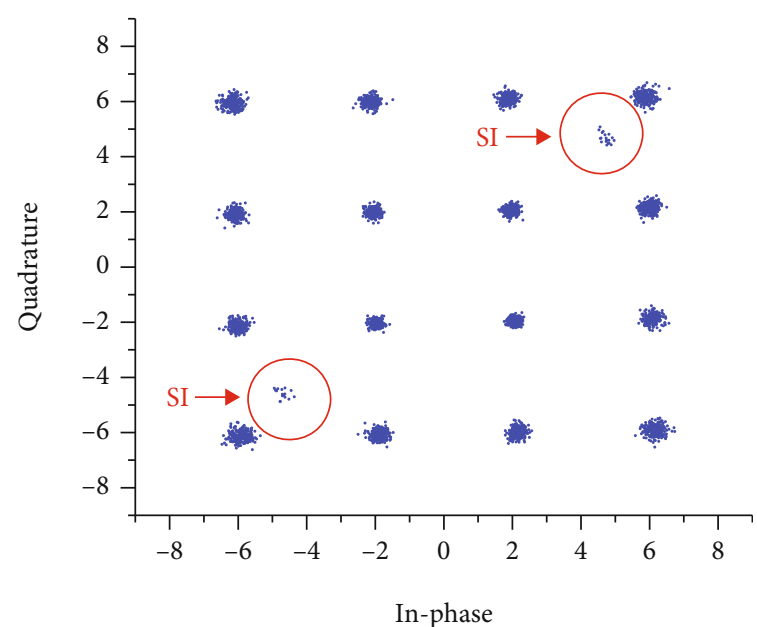

FIgURE 4: The constellation of reference signal after demodulation (one frame).

4QAM, 16QAM, 32QAM, and 64QAM constellations. Both the BPSK and 16QAM constellations can be seen in Figure 4, in which all the constellation points are focused.

After constellation demapping and deinterleave, we make a hard decision to recover the transmitted bit sequence. Finally, the recovered bit sequence is processed by the operations of interleave, constellation mapping, OFDM modulation, framing, and shaping filtering, which are the same as the transmitter, to reconstruct the pure reference signal.

4.2. Target Detection. The DJI Mavic $2 \mathrm{UAV}$, which has unfold dimensions of $322 \mathrm{~mm} \times 242 \mathrm{~mm} \times 84 \mathrm{~mm}$ is used as the target to be detected in our experiments. The clutter suppression is first conducted by using the ECA-based solution with the reconstructed reference signal and one of the dualpolarized echo signals, and the result is given in Figure 5. It shows that a suppression capacity of about $30 \mathrm{~dB}$ is achieved. Since the reconstructed reference signal cannot be consistent with the clutter components in terms of frequency deviation and time delay variation, the residual signal still has some noise components.

The decimation filter and fast Fourier transform (FFT) are used after clutter suppression to realize the CAF operation as (17). The detailed schematic is shown in Figure 6.

The obtained bistatic Rang-Doppler (RD) map after the CAF processing is shown in Figure 7. As can be seen, there is an obvious target that the bistatic range equals $119.05 \mathrm{~m}$ and the bistatic Doppler frequency equals $35 \mathrm{~Hz}$.

4.3. Polarization Diversity for Clutter Suppression. In the clutter signal, the polarization angle of the DPS component is different from that of the multipath components. Therefore, we cannot suppress every component by the way of polarization diversity.

We evaluate the power of the polarization synthesized signal as (28). When $-180^{\circ} \leq \theta \leq 180^{\circ}$ and $-180^{\circ} \leq \varphi \leq 180^{\circ}$, the obtained signal power is shown in Figure 8. For different polarization parameter pairs, the power of the synthesized signal can differ by larger than $10.5 \mathrm{~dB}$. 


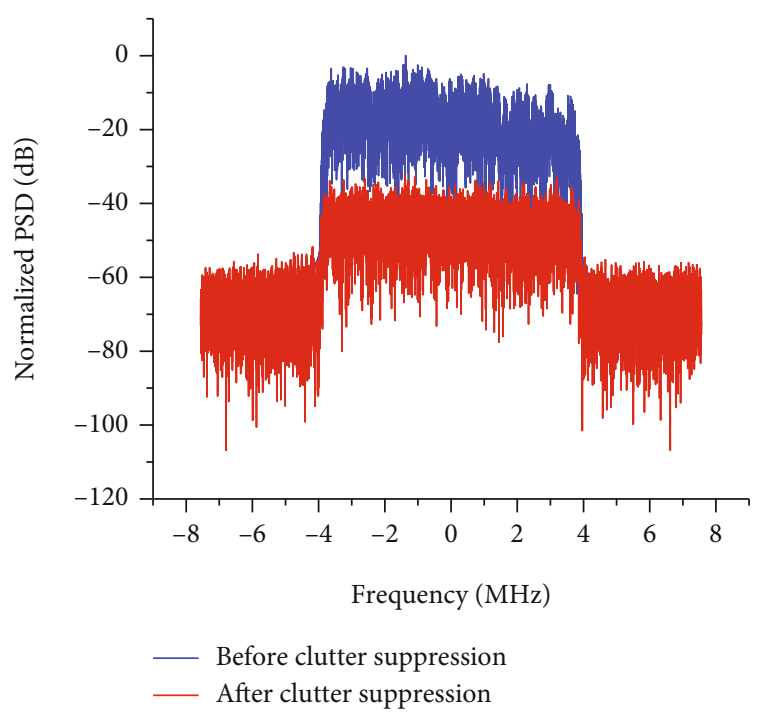

FIGURE 5: PSD comparison: before and after clutter suppression.

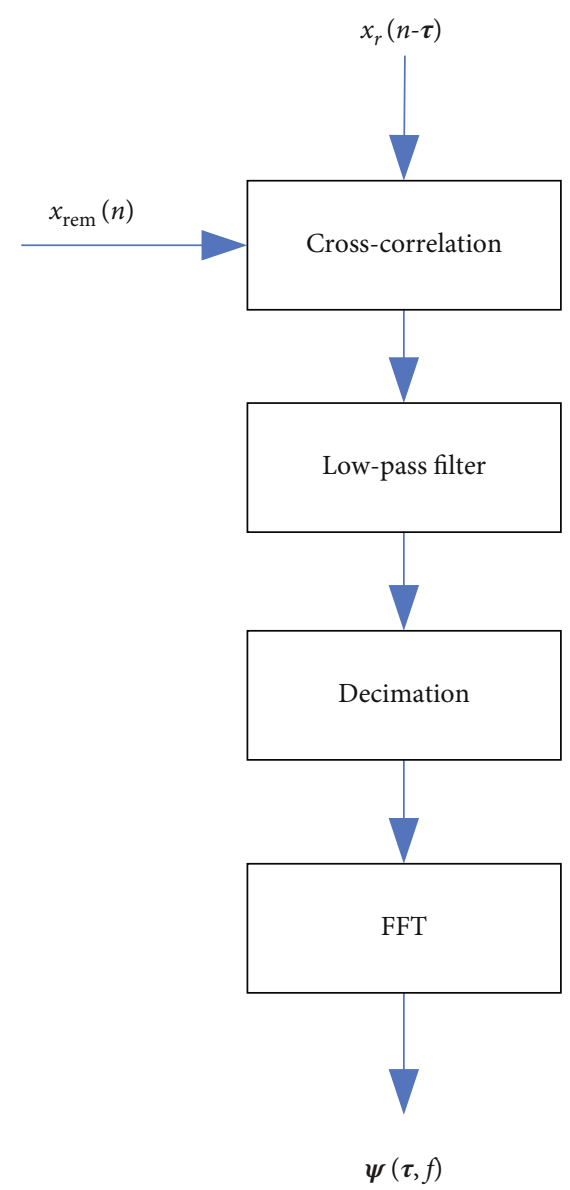

FIgURE 6: CAF processing schematic.

There are four maximums and four minimums in the range of $\left[-180^{\circ}, 180^{\circ}\right]$ due to the symmetry of trigonometric functions. Then, we narrow the range to $\left[-90^{\circ}, 90^{\circ}\right]$ and obtain the partial but complete results shown in Figure 9. In this range, the synthesized signal gets its maximum value with the parameter pair $(\theta, \varphi)$ at $\left(28^{\circ},-32^{\circ}\right)$ while its mini-

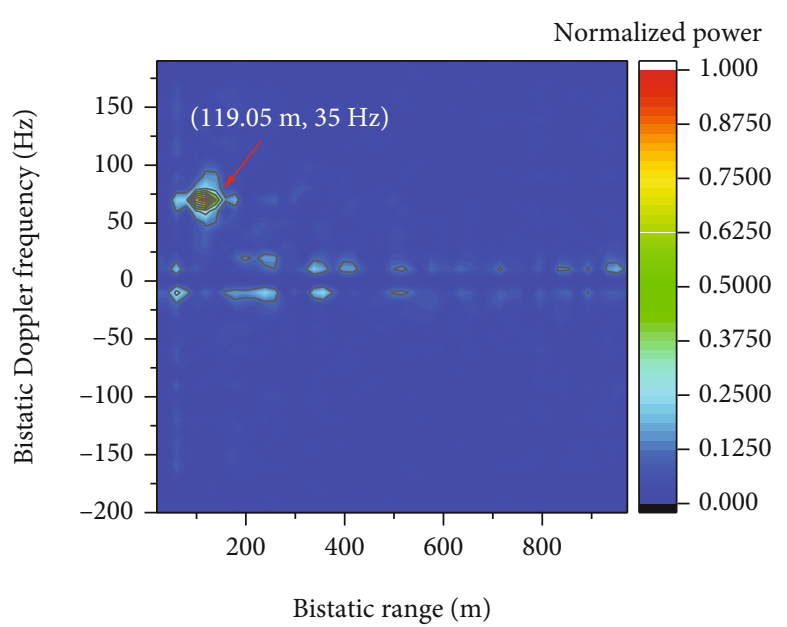

Figure 7: RD map after CAF processing.

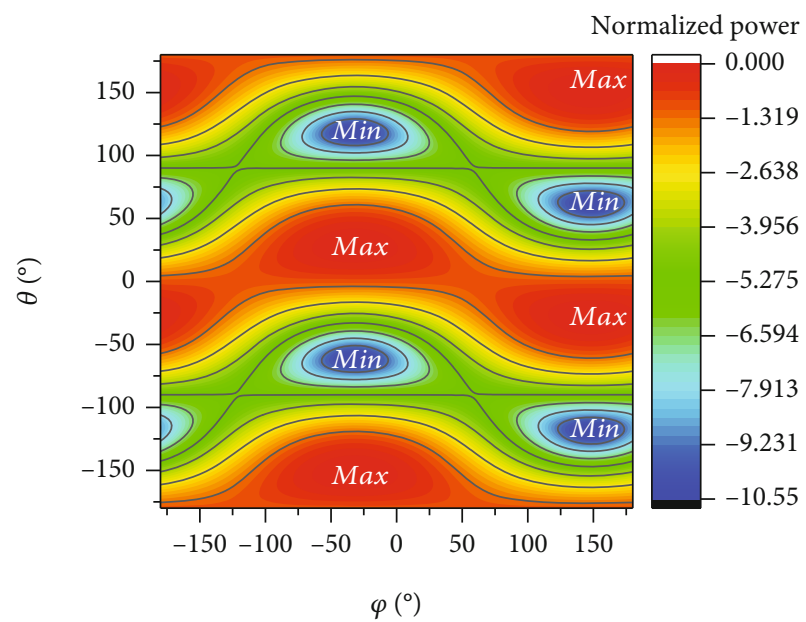

FIgURE 8: Normalized power $(\mathrm{dB})$ of the polarization synthesized signal for $-180^{\circ} \leq \theta \leq 180^{\circ}$ and $-180^{\circ} \leq \varphi \leq 180^{\circ}$.

mum value is at $\left(-62^{\circ},-32^{\circ}\right)$. As a result, the value of $-32^{\circ}$ is the estimation of the phase difference between the two orthogonal channels, and the estimated value of $28^{\circ}$ is treated as the equivalent polarization angle of the composite clutter signal. The optimal polarization angle used for clutter suppression has a value of $-62^{\circ}$ in our experiments.

4.4. Polarization Diversity for Target Detection. The target detection performance under different polarization angles is also evaluated in our experiments. Since different polarization angles may result in different residual noise components by the ECA-based solution, the SNR of the RD map defined is used in the evaluation:

$$
\mathrm{SNR}_{t}=\frac{\left|\psi\left(\tau_{t}, f_{t}\right)\right|}{\operatorname{mean}_{\tau, f}|\psi(\tau, f)|} .
$$

The obtained normalized $\mathrm{SNR}_{t}$ map is shown in Figure 10. 


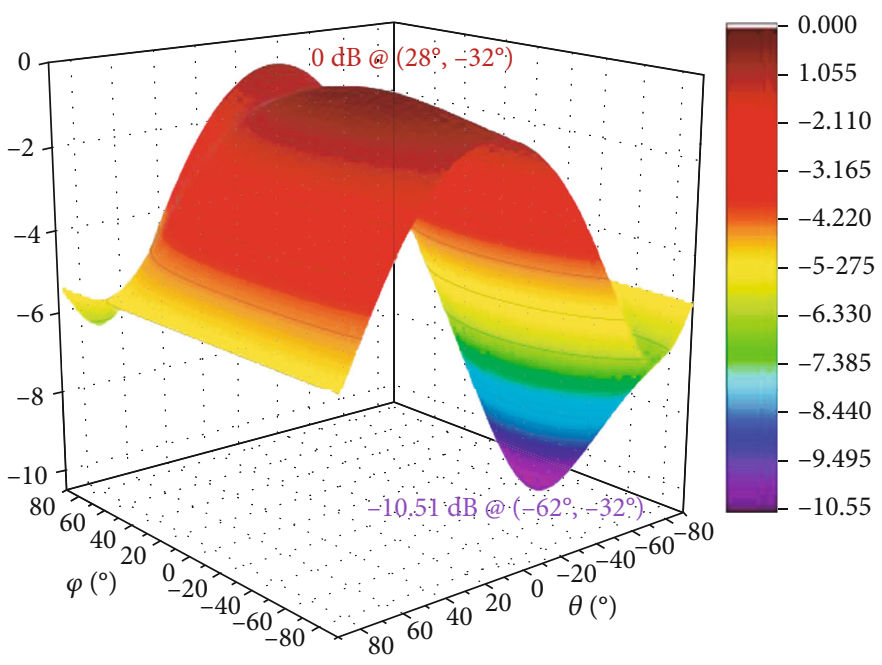

FIGURE 9: Normalized power (dB) of the polarization synthesized signal for $-90 \leq \theta \leq 90^{\circ}$ and $-90^{\circ} \leq \varphi \leq 90^{\circ}$.

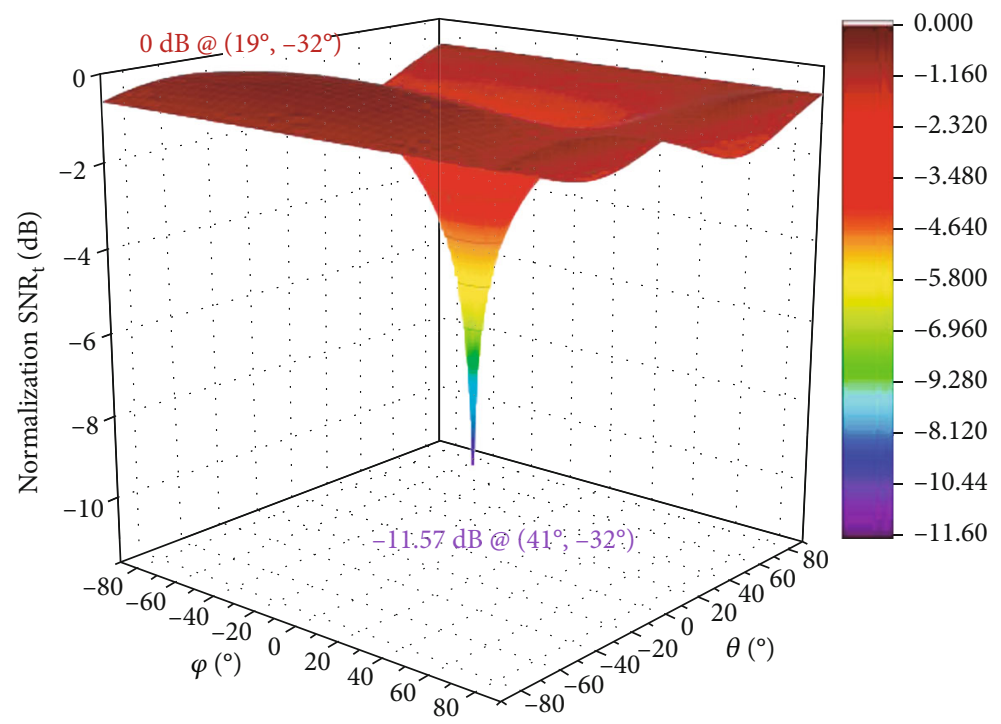

FIGURE 10: Normalized $\mathrm{SNR}_{t}(\mathrm{~dB})$ map for $-90^{\circ} \leq \theta \leq 90^{\circ}$ and $-90^{\circ} \leq \varphi \leq 90^{\circ}$.

It shows that $\mathrm{SNR}_{t}$ gets its maximum value with the parameter pair $(\theta, \varphi)$ at $\left(-49^{\circ},-32^{\circ}\right)$ while its minimum value at $\left(41^{\circ},-32^{\circ}\right)$, and the difference between the maximum and minimum is larger than $11.5 \mathrm{~dB}$. As the phase difference between the two orthogonal channels is only related to the receiver, it can be seen that the value of $\varphi$ for the extremum in Figure 10 is the same as that in Figure 9. However, the polarization angle of $-49^{\circ}$ for the maximum of $\mathrm{SNR}_{t}$ is inconsistent with the optimal value of $-62^{\circ}$ for clutter suppression. As a result, it proves that clutter suppression based on the polarization diversity technology may result in performance loss for the target detection due to the randomness of the polarization angle difference.

Table 1 shows the obtained normalized $\mathrm{SNR}_{t}$ with several polarization angles we are interested in. We only use one of the dual-polarization echo signals in the case of $\theta=0^{\circ}$. As the noises in the two orthogonal polarized channels are not
TABLE 1: Comparison of SNR .

\begin{tabular}{lcc}
\hline$\theta$ & $\varphi$ & Normalized $\mathrm{SNR}_{t}(\mathrm{~dB})$ \\
\hline $0^{\circ}$ & $0^{\circ}$ & -1.22287 \\
$28^{\circ}$ & $-32^{\circ}$ & -3.19481 \\
$-62^{\circ}$ & $-32^{\circ}$ & -0.58790 \\
$-49^{\circ}$ & $-32^{\circ}$ & 0 \\
\hline
\end{tabular}

correlated with each other, the random noise components will be superimposed after polarization synthesis. In addition to the random noise, the clutter signal and target echo signal in the synthesized signal are affected dramatically by changing the polarization angle parameter. Only when the polarization angle adopted in the polarization synthesis matches the actual signal polarization angle, the SNR of the synthesized signal can be maximized. Table 1 shows that 


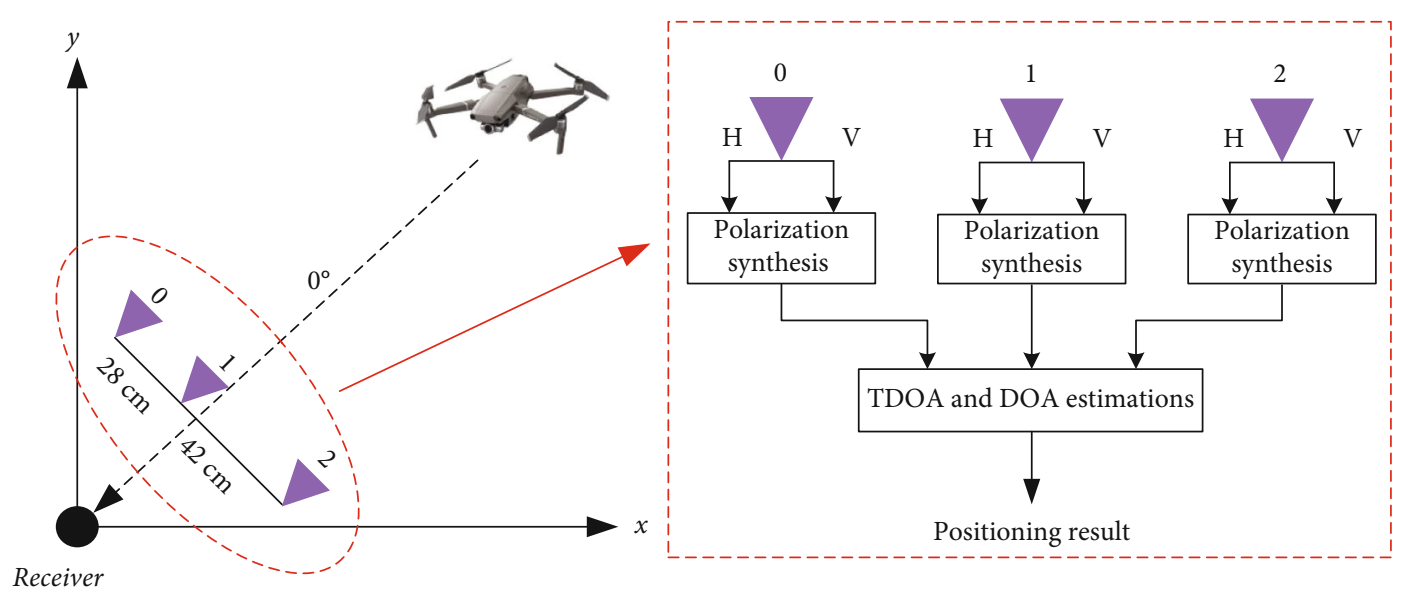

Figure 11: Array configuration of the 2-D interferometer.

polarization matching has a performance improvement of about $1.22 \mathrm{~dB}$ compared with single-polarization reception in our experiments.

As the stability of the ECA-based clutter suppression algorithm is related to the calculating precision of matrix operation, the effect of clutter suppression will not change with the amplitude of the clutter signal in a certain range. The algorithm always works within the stable range in our experiments, and the clutter signal can be well suppressed at all the polarization angles. Although there is a performance improvement of up to $2.61 \mathrm{~dB}$ compared to $28^{\circ}$ at $-62^{\circ}$, we need to point out that this is not the reason for better clutter suppression; the theoretical reason is that this polarization angle is closer to the actual polarization angle of $-49^{\circ}$. When the clutter signal is too strong for the ECA algorithm to work normally, polarization diversity is an effective way to increase the additional capacity for clutter suppression. Though there may be performance loss for target detection, it is worthful for the normal operation of the system.

4.5. Target Localization. A small antenna array which consists of three elements is exploited to estimate the DOA of the UAV echo. Figure 11 shows the configuration of the antenna array, which consists of three antenna elements. Each antenna element is orthogonally dual-polarized as shown in the figure, where $\mathrm{H}$ represents horizontal polarization and $\mathrm{V}$ represents vertical polarization. The spacing is $28 \mathrm{~cm}$ for Element 0 and Element 1 , while it is $42 \mathrm{~cm}$ for Element 1 and Element 2. Since a virtual spacing of $14 \mathrm{~cm}$ can be constructed by this nonuniform linear array, which is below the half-wavelength of about $20 \mathrm{~cm}$ in our experiments, it will not lead to the cyclically ambiguous problem in DOA estimation. For each dual-polarized element, we conduct polarization synthesis, clutter suppression, and TDOA estimation separately. The three processing results are further used for DOA estimation. The positioning result of the target is finally obtained after both TDOA and DOA estimations.

The essence of the 2-D interferometer direction finding is to determine the direction of the signal source by the phase difference formed by the received signal on different antenna elements. The wave-path difference between the two antenna

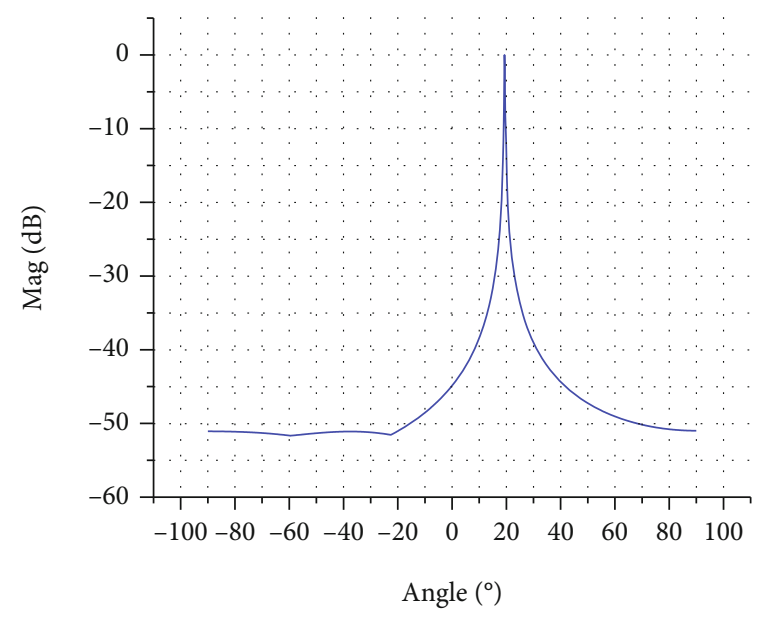

FIGURE 12: DOA output of the 2-D interferometer.

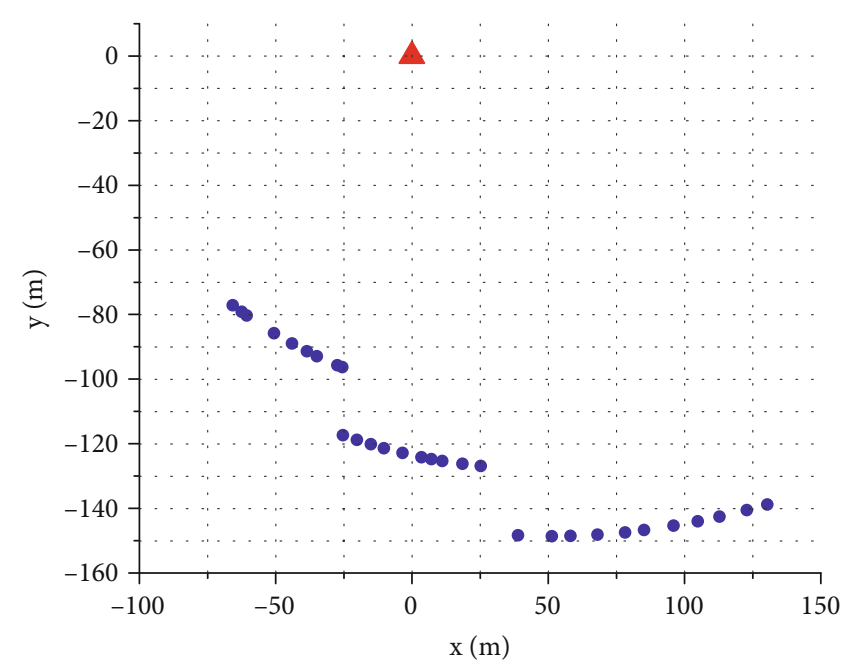

- Receiver
- UAV

FIGURE 13: Positioning result of the UAV with the combination of TDOA and DOA. 
elements will cause the phase difference. Though the polarization angle is random, it is not instantaneous. As a result, we use the same value for the parameter of $\theta$ in polarization synthesis of all the three dual-polarized antenna elements. Since the parameter of $\varphi$ is mainly related to different channels of the receiver, we use the premeasured values for it. We search optimal DOA estimation between $-90^{\circ}$ and $90^{\circ}$. The result of the direction finding at a certain moment is shown in Figure 12, which shows an optimal estimation of about $20^{\circ}$.

The combination of TDOA and DOA as (25) is adopted to locate the UAV. Figure 13 shows the positioning result of the UAV in a certain continuous time, which is consistent with its actual flight path in the experiments. The two obvious discontinuous sudden changes in Figure 13 are caused by the range resolution of the signal, which is about $39.68 \mathrm{~m}$.

Figure 13 shows that the direction finding and localization of the echo signal can still be realized after polarization synthesis, which verifies the validity of the localization algorithm in passive radar with polarization diversity reception. The positioning distance may increase significantly at some polarization angles through polarization synthesis. However, since there is only $1.22 \mathrm{~dB}$ performance improvement in target detection compared with single-polarization reception in our experiments, the positioning distance will not increase obviously.

\section{Conclusion}

We have established an experimental system to evaluate the adverse effect of polarization angle on passive radar. By analyzing the experimental data, the necessity of polarization diversity to ensure the target detection performance is revealed. The effect of polarization diversity on clutter suppression is significant; especially when the clutter signal is strong, it is necessary to increase the additional suppression capacity through polarization diversity for the normal operation of the system. However, when the clutter suppression algorithm is sufficient to suppress the clutter, the significance of the polarization diversity will lie in matching the polarization angle of the target echo signal. When the two perfectly match, the maximum SNR of the target echo signal will be achieved, and the system will have the optimal target detection performance. The target localization capacity under polarization synthesis for multichannel is also investigated. We will further study the adaptive polarization synthesis strategy to achieve a compromise between clutter suppression and target detection.

\section{Data Availability}

The data used to support the findings of this study are available from the corresponding author upon request.

\section{Conflicts of Interest}

The authors declare no conflicts of interest.

\section{Authors' Contributions}

H.Z. proposed the method, performed the experiments, and wrote the paper; L.W. designed the experiments and analyzed the data; H.Z. and M.L. made the discussion; L.W. and M.L. gave the instructional suggestions.

\section{Acknowledgments}

This work was supported in part by the Young Elite Scientists Sponsorship Program by China Association for Science and Technology (2019-2021) and in part by the National Natural Science Foundation of China (62071364).

\section{References}

[1] J. You, X. Wan, Y. Fu, and G. Fang, "Experimental study of polarisation technique on multi-FM-based passive radar," IET Radar, Sonar \& Navigation, vol. 9, no. 7, pp. 763-771, 2015.

[2] G.-H. Park, Y.-K. Seo, and H.-N. Kim, "Range-Doppler domain-based DOA estimation method for FM-band passive bistatic radar," IEEE Access, vol. 8, pp. 56880-56891, 2020.

[3] T. Martelli, F. Colone, and R. Cardinali, "DVB-T based passive radar for simultaneous counter-drone operations and civil air traffic surveillance," IET Radar, Sonar \& Navigation, vol. 14, no. 4, pp. 505-515, 2020.

[4] X. Zhang, J. Yi, X. Wan, and Y. Liu, "Reference signal reconstruction under oversampling for DTMB-based passive radar," IEEE Access, vol. 8, pp. 74024-74038, 2020.

[5] M. Wielgo, P. Krysik, K. Klincewicz, L. Maslikowski, S. Rzewuski, and K. Kulpa, "Doppler only localization in GSM-based passive radar," in in Proc. IEEE Int. Radar Conf, pp. 1-6, 2014.

[6] R. S. A. R. Abdullah, A. A. Salah, N. H. A. Aziz, and N. E. A. Rasid, "Vehicle recognition analysis in LTE based forward scattering radar," in in Proc. IEEE Radar Conf., pp. 1-5, 2016.

[7] T. Martelli, F. Colone, and P. Lombardo, "First experimental results for a WiFi-based passive forward scatter radar," in in Proc. IEEE Radar Conf., pp. 1-6, 2016.

[8] W. Li, R. J. Piechocki, K. Woodbridge, C. Tang, and K. Chetty, "Passive WiFi radar for human sensing using a stand-alone access point," IEEE Transactions on Geoscience and Remote Sensing, vol. 59, no. 3, pp. 1986-1998.

[9] Q. Wang, C. Hou, and Y. Lu, "An experimental study of WiMAX-based passive radar," IEEE Transactions on Microwave Theory and Techniques, vol. 58, no. 12, pp. 3502-3510, 2010.

[10] T. Higgins, T. Webster, and E. L. Mokole, "Passive multistatic radar experiment using WiMAX signals of opportunity. Part 1: signal processing," IET Radar, Sonar \& Navigation, vol. 10, no. 2, pp. 238-247, 2016.

[11] T. Webster, T. Higgins, and E. L. Mokole, "Passive multistatic radar experiment using WiMAX signals of opportunity. Part 2: multistatic velocity backprojection," IET Radar, Sonar \& Navigation, vol. 10, no. 2, pp. 248-255, 2016.

[12] F. Santi, D. Pastina, and M. Bucciarelli, "Experimental demonstration of ship target detection in GNSS-based passive radar combining target motion compensation and track-beforedetect strategies," Sensors, vol. 20, no. 3, p. 599, 2020. 
[13] O. Cabrera, C. Bongioanni, F. Filippini, O. Sarabakha, F. Colone, and P. Lombardo, "Detecting drones and human beings with DVB-S based COTS passive radar for shortrange surveillance," in in Proc. IEEE Int. Radar Conf, pp. 3742, 2020.

[14] M. Conti, C. Moscardini, and A. Capria, "Dual-polarization DVB-T passive radar: experimental results," in in Proc. IEEE Radar Conf, pp. 1-5, 2016.

[15] F. Colone and P. Lombardo, "Polarimetric passive coherent location," IEEE Transactions on Aerospace and Electronic Systems, vol. 51, no. 2, pp. 1079-1097, 2015.

[16] F. Colone and P. Lombardo, "Non-coherent adaptive detection in passive radar exploiting polarimetric and frequency diversity," IET Radar, Sonar \& Navigation, vol. 10, no. 1, pp. 1523, 2016.

[17] Y. Yi, X. Wan, J. Yi, and X. Cao, "Polarization diversity technology research in passive radar based on subcarrier processing," IEEE Sensors Journal, vol. 19, no. 5, pp. 1710-1719, 2019.

[18] L. Wan, K. Liu, Y.-C. Liang, and T. Zhu, "DOA and polarization estimation for non-circular signals in 3-D millimeter wave polarized massive MIMO systems," IEEE Trans. Wirel. Commun, 2021.

[19] M. Malanowski, Signal Processing for Passive Bistatic Radar, Artech House, Norwood, MA, USA, 2019.

[20] P. Zhang, Y. Wu, J. Wang, and J. Qiao, "Real-time signal processing for FM-based passive bistatic radar using GPUs," in in Proc. IEEE Int. Conf. Digital Signal Processing, pp. 536-540, 2014.

[21] A. Noroozi and M. A. Sebt, "Target localization from bistatic range measurements in multi-transmitter multi-receiver passive radar," IEEE Signal Processing Letters, vol. 22, no. 12, pp. 2445-2449, 2015.

[22] M. Malanowski and K. Kulpa, "Two methods for target localization in multistatic passive radar," IEEE Transactions on Aerospace and Electronic Systems, vol. 48, no. 1, pp. 572-580, 2012.

[23] M. Liu, G. Liao, N. Zhao, H. Song, and F. Gong, "Data-driven deep learning for signal classification in industrial cognitive radio networks," IEEE Transactions on Industrial Informatics, vol. 17, no. 5, pp. 3412-3421, 2021.

[24] Y. Zhao, D. Hu, Y. Zhao, Z. Liu, and C. Zhao, "Refining inaccurate transmitter and receiver positions using calibration targets for target localization in multi-static passive radar," Sensors, vol. 19, no. 15, 2019.

[25] M. Ummenhofer, M. Kohler, J. Schell, and D. W. O'Hagan, "Direction of arrival estimation techniques for passive radar based 3D target localization," in in Proc. IEEE Radar Conf, pp. 1-6, 2019.

[26] L. Wan, Y. Sun, L. Sun, Z. Ning, and J. J. P. C. Rodrigues, "Deep learning based autonomous vehicle super resolution DOA estimation for safety driving," in IEEE Trans. Intell. Transp. Syst, 2020.

[27] G. Bournaka, J. Heckenbach, A. Baruzzi, D. Cristallini, and H. Kuschel, "A two stage beamforming approach for low complexity CFAR detection and localization for passive radar," in in Proc. IEEE Radar Conf., pp. 1-4, 2016.

[28] M. Liu, K. Yang, N. Zhao, Y. Chen, H. Song, and F. Gong, "Intelligent signal classification in industrial distributed wireless sensor networks based industrial internet of things," IEEE Transactions on Industrial Informatics, vol. 17, no. 7, pp. 4946-4956, 2021.
[29] J. H. Huang, J. L. Garry, G. E. Smith, and C. J. Baker, "Array based passive radar target localization," in in Proc. IEEE Radar Conf, pp. 1-6, 2016.

[30] L. Wan, L. Sun, K. Liu, X. Wang, Q. Lin, and T. Zhu, “Autonomous vehicle source enumeration exploiting non-cooperative UAV in software defined Internet of vehicles," in IEEE Trans. Intell. Transport. Syst, 2020.

[31] P. Falcone, F. Colone, A. Macera, and P. Lombardo, "Twodimensional location of moving targets within local areas using WiFi-based multistatic passive radar," IET Radar, Sonar \& Navigation, vol. 8, no. 2, pp. 123-131, 2014. 